\title{
Prediction of anastomotic leakage after anterior rectal resection
}

\author{
Shubang Cheng ${ }^{1}$, Bolin He${ }^{2}$, Xueyi Zeng ${ }^{3}$
}

\begin{abstract}
Objective: Anastomotic Leakage (AL) is one of the most common complications after resection of rectal cancer. Recognition of the incidence and risk factors related to AL is important. This study aimed develops a model that can predict anastomotic leakage after anterior rectal resection.

Methods: Data from 188 patients undergoing anterior resection of rectal cancer were collected for retrospective analysis. Patients were randomly divided in the development set and validation set at a 1:1 ratio. We first included age, sex, preoperative chemoradiotherapy, tumor size, degree of tumor differentiation, stage, TNM stage, lymph vascular invasion, distance, anastomotic method, diabetes, intraoperative time, intraoperative bleeding and smoking as candidates for variable selection with a LASSO method. A ROC curve was constructed with the validation set to assess the accuracy of the prediction model.

Results: AL occurred in 20 of 188 patients (10.6\%). Preoperative chemoradiotherapy $(p=0.04)$, medium degree of tumor differentiation $(p=0.04)$, anastomotic method $(p<0.01)$, intraoperative bleeding $\geq 400 \mathrm{ml}$ $(p<0.01)$, smoking $(p<0.01)$, diabetes $(p<0.01)$ were significantly related to AL. The area under the ROC curve of the prediction model is 0.952 .

Conclusions: This study developed a model that can predict anastomotic leakage after anterior rectal resection, which may aid the selection of preventive ileostomy and postoperative management.
\end{abstract}

KEYWORDS: Anastomotic leakage, LASSO, Prediction, Rectal cancer.

doi: https://doi.org/10.12669/pjms.35.3.252

How to cite this:

Cheng S, He B, Zeng X. Prediction of anastomotic leakage after anterior rectal resection. Pak J Med Sci. 2019;35(3):830-835.

doi: https://doi.org/10.12669/pjms.35.3.252

This is an Open Access article distributed under the terms of the Creative Commons Attribution License (http://creativecommons.org/licenses/by/3.0), which permits unrestricted use, distribution, and reproduction in any medium, provided the original work is properly cited.

1. Dr. Shubang Cheng, MD,

2. Dr. Bolin $\mathrm{He}, \mathrm{MD}$,

Department of Gastrointestinal,

People's Hospital of Longhua District,

Affiliated Hospital of Guangdong Medical University,

Shenzhen, Guangdong Province, China.

3. Dr. Xueyi Zeng, MD,

1,3: Department of Gastrointestinal,

People's Hospital of Longhua District,

Affiliated Hospital of Guangdong Medical University,

Shenzhen, Guangdong Province,

China.

Correspondence:

Dr. Shu-bang Cheng,

People's Hospital of Longhua District,

Affiliated Hospital of Guangdong Medical University,

Shenzhen, Guangdong Province, 518109,

China.

Email: shubangcheng_sz@sina.com

* Received for Publication:

December 24, 2018

* Accepted for Publication:

April 8, 2019

\section{INTRODUCTION}

Rectal cancer is the third most common malignant tumor in the world. The incidence in China has been increasing at a rate of $4.2 \%$ per year. ${ }^{1}$ Surgery has been a common treatment approach for rectal cancer. But anastomotic leakage (AL) is one of the most frequent complications after the resection of rectal tumors. The incidence of postoperative AL in rectal cancer is $3 \%-13 \% .^{2,3}$ It can cause serious consequences such as pelvic abscesses, peritonitis, sepsis, septic shock and even death. Moreover, AL is also the main course of long-term complications such as postoperative anastomotic stenosis and bowel dysfunction. Although significant progress has been made in the stapler, operation technique 
and perioperative management in recent ten years, anastomotic leakage remains a major issue in clinical practice.

Many factors ${ }^{4}$ are believed to affect the anastomosis, such as age, sex, level of anastomosis, ASA score, BMI, steroid treatment, preoperative chemoradiotherapy, tumor stage, tumor size, operative time, number of stapler firings, weight loss, malnutrition, fluid/ electrolyte disorders, alcohol consumption, intraoperative transfusions/blood loss, smoking and diabetes but the pathogenesis remains unclear, which brings difficulties to accurately predict AL in rectal cancer. Thus colorectal surgeons may have to empirically use preventive colostomy for patients.

In this study, we aimed to develop a risk model to predict the occurrence of postoperative $\mathrm{AL}$ and aid the proper selection of preventive ileostomy.

\section{METHODS}

This study was approved by the Institutional Review Board (IRB) of People's Hospital of Longhua District (No. LW-20170301-003-01). The IRB waived the written informed consent from patients since this study was carried out retrospectively.

Patients with rectal cancer who underwent surgical treatment in our hospital from 2010 to 2016 were enrolled. Rectal cancer was defined as a tumor located at $15 \mathrm{~cm}$ or less from the anal verge, as determined by endoscopy and/or digital rectal examination. The inclusion criteria were patients undergoing DIXON surgery without preventive colostomy.

\section{Exclusion criteria were:}

1. Patients with colon cancer at the descending colon or above the descending colon

2. Patients undergoing Miles surgery

3. Patients undergoing Hartman surgery

4. Patients undergoing transanal or transsacral local excision of rectal cancer.

Finally, 188 patients were included, and amongst them 20 had postoperative anastomotic leakage. The diagnosis of AL was based on clinical manifestations and imaging results.

Operation: For middle and low rectal cancer, the operation was strictly restricted to the procedure of total mesorectal excision (TME). Tumor-specific mesorectal excision (TSME) was performed for the upper rectal cancer and when the mesorectal excision level was $5 \mathrm{~cm}$ from the lower edge of the tumor. ${ }^{5,6}$ The mode of anastomosis includes stapled anastomosis and manual anastomosis. After the anastomosis, the leak test was performed. The conventional indwelling duration of drainage tube was 7-15 days.

Statistical analysis: Statistical analysis was conducted in R. Mean (standard deviation) and frequency (percentage) were summarized by anastomotic states for continuous and categorical variables, respectively. Chi-square tests or Fisher Exact tests were used for comparison of categorical variables and the Wilcoxon tests were used for comparison of real-value variables between the $\mathrm{AL}$ and non-AL groups. Half of the patients in each group were randomly selected as a training set to develop a prediction model with the LASSO method. ${ }^{7}$ The remaining cases were used as a validation set.

\section{RESULTS}

A total of 188 patients were included and 20 patients including 15 males and four females had postoperative AL with an incidence rate of $10.6 \%$. The patient-related variables, tumorrelated variables, and surgery-related variables were selected for univariate analysis (Table-I) and the degree of tumor differentiation, the distance between the lower edge of the tumor and the anal margin, anastomotic method, diabetes, intraoperative time, intraoperative bleeding and smoking history were significantly associated with AL $(p<0.05)$.

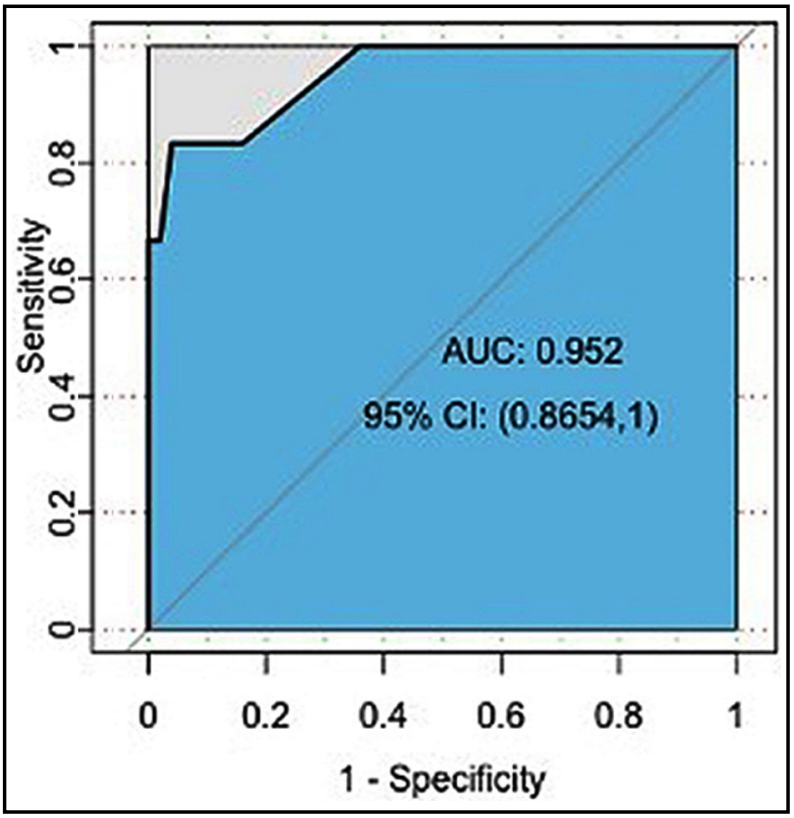

Fig.1: The ROC for occurrence of anastomotic leak prediction model. 
Table-I: Univariate analysis of risk factors for anastomotic leakage.

\begin{tabular}{|c|c|c|c|c|}
\hline Variable & Overall & $\begin{array}{c}\text { No Anastomotic Leak } \\
(n=168)\end{array}$ & $\begin{array}{c}\text { Anastomotic Leak } \\
(n=20)\end{array}$ & $P$-value \\
\hline Age & $61.11 \pm 14.02$ & $61.66(13.71)$ & $56.45(16.06)$ & 0.2152 \\
\hline \multicolumn{5}{|l|}{ Sex } \\
\hline Female & $72(38.29)$ & $68(40.48)$ & $4(20)$ & \multirow[t]{2}{*}{0.09} \\
\hline Male & $116(61.70)$ & $100(59.52)$ & $16(80)$ & \\
\hline \multicolumn{5}{|c|}{ Preoperative chemoradiotherapy } \\
\hline $\mathrm{N}$ & $174(92.55)$ & $156(92.86)$ & $18(90)$ & \multirow[t]{2}{*}{0.648} \\
\hline Y & $14(7.45)$ & $12(7.14)$ & $2(10)$ & \\
\hline \multicolumn{5}{|l|}{ Tumor size } \\
\hline$<4 \mathrm{~cm}$ & $72(38.29)$ & $66(39.29)$ & $6(30)$ & \multirow[t]{2}{*}{0.5726} \\
\hline$\geq 4 \mathrm{~cm}$ & $116(61.70)$ & $102(60.71)$ & $14(70)$ & \\
\hline \multicolumn{5}{|c|}{ Degree of tumor differentiation } \\
\hline High differentiation & $11(5.85)$ & $7(4.17)$ & $4(20)$ & \multirow[t]{3}{*}{ 8.24E-06 } \\
\hline Medium differentiation & $161(85.6)$ & $152(90.48)$ & $9(45)$ & \\
\hline Low differentiation & $16(8.51)$ & $9(5.36)$ & $7(35)$ & \\
\hline \multicolumn{5}{|l|}{ Stage } \\
\hline $\mathrm{T} 1+\mathrm{T} 2$ & $36(19.15)$ & $34(20.24)$ & $2(10)$ & \multirow{2}{*}{0.375} \\
\hline $\mathrm{T} 3+\mathrm{T} 4$ & $152(80.85)$ & $134(79.76)$ & $18(90)$ & \\
\hline \multicolumn{5}{|l|}{ TNM stage } \\
\hline 0 & $1(5)$ & $1(0.6)$ & 0 & \multirow[t]{5}{*}{0.1422} \\
\hline I & $30(15.96)$ & $29(17.26)$ & $1(5)$ & \\
\hline II & $64(34.04)$ & $60(35.71)$ & $4(20)$ & \\
\hline III & $81(43.09)$ & $67(39.88)$ & $14(70)$ & \\
\hline IV & $12(6.38)$ & $11(6.55)$ & $1(5)$ & \\
\hline \multicolumn{5}{|l|}{ Lymph vascular invasion } \\
\hline $\mathrm{N}$ & $97(51.60)$ & $88(52.38)$ & $9(45)$ & \multirow[t]{2}{*}{0.6982} \\
\hline Y & $91(48.40)$ & $80(47.62)$ & $11(55)$ & \\
\hline \multicolumn{5}{|l|}{ Distance } \\
\hline$<7 \mathrm{~cm}$ & $51(27.13)$ & $38(22.62)$ & $13(65)$ & \multirow[t]{2}{*}{0.0001674} \\
\hline$\geq 7 \mathrm{~cm}$ & $137(72.87)$ & $130(77.38)$ & $7(35)$ & \\
\hline \multicolumn{5}{|l|}{ Anastomotic method } \\
\hline Hand-sewn & $3(1.60)$ & $1(0.6)$ & $2(10)$ & \multirow[t]{2}{*}{0.03033} \\
\hline Stapler & $185(98.40)$ & $167(99.40)$ & $18(90)$ & \\
\hline \multicolumn{5}{|l|}{ Diabetes } \\
\hline $\mathrm{N}$ & $156(82.98)$ & $149(88.69)$ & $7(35)$ & \multirow[t]{2}{*}{$3.29 \mathrm{E}-07$} \\
\hline Y & $32(17.02)$ & 19(11.31) & $13(65)$ & \\
\hline \multicolumn{5}{|l|}{ Intraoperative time } \\
\hline$<3.5 \mathrm{~h}$ & $136(72.34)$ & $127(75.60)$ & $9(45)$ & \multirow[t]{2}{*}{0.006974} \\
\hline$\geq 3.5 \mathrm{~h}$ & $52(27.66)$ & $41(24.40)$ & $11(55)$ & \\
\hline \multicolumn{5}{|l|}{ Intraoperative bleeding } \\
\hline$<400 \mathrm{ml}$ & $170(90.43)$ & $162(96.43)$ & $8(40)$ & \multirow[t]{2}{*}{ 6.34E-10 } \\
\hline$\geq 400 \mathrm{ml}$ & $18(9.57)$ & $6(3.57)$ & $12(60)$ & \\
\hline Smoking & & & & \\
\hline $\mathrm{N}$ & $146(77.66)$ & $138(82.14)$ & $8(40)$ & 0.0001219 \\
\hline Y & $42(22.34)$ & $30(17.86)$ & $12(60)$ & \\
\hline
\end{tabular}

Data are presented as mean \pm SD or No. (\%);

Distance: distance between the lower edge of tumor and the anal margin. 
Shubang Cheng et al.

Table-II: Multivariable analysis of risk factors for anastomotic leakage.

\begin{tabular}{lccc}
\hline Factors & OR & Confidence interval & P-value \\
\hline Preoperative chemoradiotherapy $(\mathrm{Y})$ & 21.01865 & $1.17 \sim 784.88$ & 0.045 \\
Degree of tumor differentiation (Medium) & 0.02 & $1.67 \mathrm{E}-04 \sim 0.72$ & 0.040 \\
Degree of tumor differentiation (Low) & 0.24 & $1.31 \mathrm{E}-03 \sim 20.63$ & 0.542 \\
Anastomotic method (Stapler) & $5.74 \mathrm{E}-04$ & $6.36 \mathrm{E}-07 \sim 0.08$ & 0.007 \\
Distance $(\geq 7 \mathrm{~cm})$ & 0.30 & $0.02 \sim 3.11$ & 0.321 \\
Intraoperative bleeding $(\geq 400 \mathrm{ml})$ & 124.39 & $10.76 \sim 5403.72$ & 0.001 \\
Smoking $(\mathrm{Y})$ & 179.80 & $14.03 \sim 8337.25$ & $<0.001$ \\
Diabetes $(\mathrm{Y})$ & 301.02 & $23.70 \sim 1722.13$ & $<0.001$ \\
\hline
\end{tabular}

Then the factors including preoperative chemoradiotherapy, degree of tumor differentiation, anastomotic method, distance, intraoperative bleeding, smoking and diabetes were selected for multivariable analysis (Table-II). The significant risk factors for AL were preoperative chemoradiotherapy $(\mathrm{p}=0.04)$, medium degree of tumor differentiation $(p=0.04)$, anastomotic method $(p<0.01)$, intraoperative bleeding more than $400 \mathrm{ml}(p<0.01)$, smoking $(\mathrm{p}<0.01)$, and diabetes $(\mathrm{p}<0.01)$. The validation set was used to construct the ROC curve (Fig.1) and the area under the curve (AUC) of the ROC was 0.952 , indicating a good performance of the prediction model.

\section{DISCUSSION}

AL can potentially affect short period recurrences and long-term survival. ${ }^{8,9}$ Therefore, in this study, we aimed to develop a risk model to predict the occurrence of postoperative anastomotic leakage, to guide the surgeons to select the preventive ileostomy in a more scientific and standardized way.

Despite the conspicuous progress in rectal cancer surgery, about $10.6 \%$ of the patients still have AL in our study, within the previously reported range of incidence rate (3\%-13\%). To select appropriate predictors, adaptive LASSO (least absolute shrinkage and selection operator) was used. LASSO method was originally developed for variable selection in building regression models and, Zou upgraded the LASSO to adaptive LASSO so that the selected model will be very close to the true models (Oracle property) as the sample size increases. ${ }^{7}$ The adaptive LASSO employs the appropriate penalty term to allow some of the coefficients to be estimated as zeros (variable exclusion) which is called sparsity regression. Unlike the stepwise regression using multiple steps and subjectively selected cutoff value of the p-value for exclusion, the selection of the predictors using adaptive
LASSO is simultaneously achieved and the tuning parameter selection is data-driven.

We found that preoperative chemoradiotherapy, diabetes, smoking, the amount of intraoperative bleeding, anastomotic method, and degree of tumor differentiation were independent AL risk factors after resection of rectal cancer. Consistently, preoperative chemoradiotherapy for rectal cancer has been previously reported as a risk factor for AL. ${ }^{10}$

The histological changes of blood vessels and epithelial tissue induced by preoperative chemoradiotherapy give rise to the destruction of mucosal inflammation and mucosal barriers ${ }^{11}$, which may lead to mucosal atrophy, fibrosis of the intestinal wall and hardening of the blood vessels, making the use of the stapler difficult and increasing the incidence of AL. But future studies are still needed to investigate the impact of preoperative chemoradiotherapy on $\mathrm{AL}$ as some reports also show that preoperative chemoradiotherapy does not affect the technical feasibility of the stapler and does not significantly increase the morbidity of postoperative complications such as AL. ${ }^{12,13}$

Smoking has been reported to affect the healing of anastomotic stoma in gastrointestinal surgery. ${ }^{14}$ Nicotine affects arterial smooth muscle and slows blood flow. Inhalation of carbon monoxide combined with hemoglobin, resulting in the ability of blood to carry oxygen down, affecting blood coagulation and tissue remodeling. Smoking may change the use of Nitrous Oxide in the blood, and produce blood vessels destruction. In terms of the blood itself, smoking can cause changes in the function of the blood circulation system, cause insufficient blood supplied and impede the healing of the wound. On the other hand, smoking may alter the mechanism of inflammation, reduce the influx of macrophages in the inflammatory response, and reduce the formation of proinflammatory factors that control adhesion and migration. Vignali A et 
al. ${ }^{2}$ reported a correlation between diabetes mellitus and AL. Diabetic patients with systemic metabolic disorder and hyperglycemia lead to large amounts of reactive oxygen species (ROS) and advanced glycation end products (AGEs) ${ }^{15}$, which damage microvascular endothelial cells, resulting in abnormal flow of microvascular endothelial cells. Lipid metabolism and glucose metabolism disorder coexist in diabetic patients. The damage mechanism of lipotoxicity on microvascular endothelial cells is the increasing production of reactive oxygen species (ROS) caused by the up regulation of the expression level of the NADPH oxidase complex active functional subunit ${ }^{16}$. The damage of the anastomotic microvessels hinders the healing of the anastomotic stoma.

Hand-sewn was also found to be a risk factor of anastomotic leakage. There was significant difference $(p<0.05)$ in AL between stapled and hand-sewn colorectal anastomosis. A Cochrane review of colorectal anastomosis after right hemicolectomy showed that the total anastomotic leakage rate in the stapler group was significantly reduced. ${ }^{17}$ An interesting subgroup analysis conducted by Friend PJ et al. ${ }^{18}$ found that there was more anastomotic leakage in hand-sewn colorectal anastomosis when analysing the anastomosis alone. Their conclusion is that stapler anastomosis seems to be more suitable for surgeons without numerous surgical experience. The double stapling technique reduces the difficulty of the operation and reduces the difficulty of anastomosis due to the inconsistent caliber of the bowel lumen at both ends. Moreover, the design of the nail spacing, the row spacing and the varus degree of the intestinal wall by the stapler was more accurate and reasonable than manual suture. It can effectively avoid overflow of intestinal contents and reduce the chance of infection around the anastomotic stoma as well as the pelvic cavity. So the incidence of anastomotic leakage can be reduced.

Wang $\mathrm{L}$ and $\mathrm{Gu} \mathrm{J}{ }^{19}$ reported the risk factor for symptomatic $\mathrm{AL}$ after anterior resection of low rectal cancer. Their results showed that blood loss more than $200 \mathrm{ml}$ was identified as independent risk factors for AL. A systematic review by McDermott FD et al. ${ }^{20}$ showed that blood loss more than $100 \mathrm{ml}$ was an independent risk factor for AL. In contract, Crombe $\mathrm{T}$ et al. ${ }^{21}$ reported that blood loss more than $500 \mathrm{ml}$ did not significantly increase the risk of AL. In our study, intraoperative bleeding more than 400 $\mathrm{ml}$ was significantly associated with the increased risk of AL. This result suggests that prophylactic stoma should be considered when the amount of bleeding is large during the operation.

Although there have been several studies ${ }^{22,23}$ reported that $\mathrm{T}$ staging is associated with the occurrence of $\mathrm{AL}$, no studies have addressed the relationship between AL and the degree of tumor differentiation. Our study shows that the degree of tumor differentiation is also a risk factor of anastomotic leakage after anterior resection of rectal cancer.

Low anastomosis is the most important risk factor for AL. ${ }^{24,25}$ In a previous study ${ }^{24}$, anal verge distance $<7 \mathrm{~cm}$ was a risk factor associated with AL occurrence. A study conducted by Vignali A et al. ${ }^{2}$ showed that the incidence of anastomotic leakage was $8 \%$ when the lower edge of the tumor was within $7 \mathrm{~cm}$ from the anal margin, and when the tumor's lower margin was more than $7 \mathrm{~cm}$ from the anal margin, it was $1 \%$. Pakkastie TE et al. ${ }^{25}$ described similar results. They also determined the difference between high and low anastomosis at a distance of $7 \mathrm{~cm}$ from the edge of the anus. The high leakage rate associated with low anastomosis is probably due to the location of the tumor was lower, and that the free range of rectal surgery is greater, which increase the difficulty of the anastomosis with increased anastomotic tension and decrease the rectal blood supply. So it affects local healing and anti-infection ability, and increases the chance of bacterial infection, resulting in an increased chance of AL. However, our study found that Distance $(\geq 7 \mathrm{~cm})$ was not protective factor for AL $(p=0.32)$. Future studies are required to investigate feasibility of the selection of $7 \mathrm{~cm}$ as a cutoff point.

Limitations of study: This was a single center study, which result in sample bias. This prediction model could not define the actual situation of AL according to current risk factors. We expect that a prospective large-sample-size and multicenter study could be conducted in future to improve the reliability and practicability of the prediction model.

\section{CONCLUSION}

We have developed a model to predict $\mathrm{AL}$ after anterior rectal resection by using the LASSO method with an AUC 0.952. Preoperative chemoradiotherapy, diabetes, smoking, intraoperative bleeding, degree of tumor differentiation and method of anastomosis are independent risk factors for AL.

Grant Support \& Financial Disclosures: None. 


\section{REFERENCES}

1. Li M, Gu J. Changing patterns of colorectal cancer in China over a period of 20 years. World J Gastroenterol. 2005;11(30):4685-4688.

2. Vignali A, Fazio VW, Lavery IC, Milsom JW, Church JM, Hull TL, et al. Factors associated with the occurrence of leaks in stapled rectal anastomoses: a review of 1,014 patients. J Am Coll Surg. 1997;185(2):105-113.

3. Matsuda K, Hotta T, Takifuji K, Yokoyama S, Oku Y, Watanabe $\mathrm{T}$, et al. Randomized clinical trial of defaecatory function after anterior resection for rectal cancer with high versus low ligation of the inferior mesenteric artery. Br J Surg. 2015;102(5):501-508. doi: 10.1002/bjs.9739.

4. Kang CY, Halabi WJ, Chaudhry OO, Nguyen V, Pigazzi A, Carmichael JC, et al. Risk factors for anastomotic leakage after anterior resection for rectal cancer. JAMA Surg. 2013;148(1):65-71. doi: 10.1001/2013.jamasurg.2.

5. Heald RJ. Rectal cancer: the surgical options. Eur J Cancer. 1995;31A(7-8):1189-1192.

6. Kim JH, Kim HY, Lee IK, Oh ST, Kim JG, Lee YS. Intraoperative double-stapled colorectal or coloanal anastomotic complications of laparoscopic low anterior resection for rectal cancer: double-stapled anastomotic complication could result in persistent anastomotic leakage. Surg Endosc. 2015;29(11):3117-3124. doi: 10.1007/s00464-014-4035-3.

7. Hui Z. The Adaptive Lasso and Its Oracle Properties. J Am Stat Assoc. 2006;101(476):1418-1429.

8. Krarup PM, Nordholm-Carstensen A, Jorgensen LN, Harling $\mathrm{H}$. Anastomotic leak increases distant recurrence and longterm mortality after curative resection for colonic cancer: a nationwide cohort study. Ann Surg. 2014;259(5):930-938. doi: 10.1097/SLA.0b013e3182a6f2fc.

9. Mirnezami A, Mirnezami R, Chandrakumaran K, Sasapu $K$, Sagar P, Finan P. Increased local recurrence and reduced survival from colorectal cancer following anastomotic leak: systematic review and meta-analysis. Ann Surg. 2011;253(5):890-899. doi: 10.1097/SLA.0b013e3182128929.

10. Qin Q, Ma T, Deng Y, Zheng J, Zhou Z, Wang H, et al. Impact of Preoperative Radiotherapy on Anastomotic Leakage and Stenosis After Rectal Cancer Resection: Post Hoc Analysis of a Randomized Controlled Trial. Dis Colon Rectum. 2016;59(10):934-942. doi: 10.1097/DCR.0000000000000665.

11. Hauer-Jensen M, Wang J, Boerma M, Fu Q, Denham JW. Radiation damage to the gastrointestinal tract: mechanisms, diagnosis, and management. Curr Opin Support Palliat Care. 2007;1(1):23-29. doi: 10.1097/SPC.0b013e3281108014.

12. Liang JT, Lai HS, Cheng KW. Technical feasibility of laparoscopic total mesorectal excision for patients with low rectal cancer after concurrent radiation and chemotherapy with bevacizumab plus FOLFOX. Surg Endosc. 2011;25(1):305-308. doi: 10.1007/s00464-010-1125-8.

13. Valero G, Lujan JA, Hernandez Q, De Las Heras M, Pellicer E, Serrano A, et al. Neoadjuvant radiation and chemotherapy in rectal cancer does not increase postoperative complications. Int J Colorectal Dis. 2003;18(6):495-499. doi: 10.1007/s00384003-0520-1.

14. Kim MJ, Shin R, Oh HK, Park JW, Jeong SY, Park JG. The impact of heavy smoking on anastomotic leakage and stricture after low anterior resection in rectal cancer patients. World J Surg. 2011;35(12):2806-2810. doi: 10.1007/s00268011-1286-1.
15. De Bock K, Georgiadou M, Schoors S, Kuchnio A, Wong BW, Cantelmo AR, et al. Role of PFKFB3-driven glycolysis in vessel sprouting. Cell. 2013;154(3):651-663. doi: 10.1016/j. cell.2013.06.037.

16. Kim JA, Montagnani M, Chandrasekran S, Quon MJ. Role of lipotoxicity in endothelial dysfunction. Heart Fail Clin. 2012;8(4):589-607. doi: 10.1016/j.hfc.2012.06.012.

17. Choy PY, Bissett IP, Docherty JG, Parry BR, Merrie AE. Stapled versus handsewn methods for ileocolic anastomoses. Cochrane Database Syst Rev. 2007(3):CD004320. doi: 10.1002/14651858.CD004320.pub2.

18. Friend PJ, Scott R, Everett WG, Scott IH. Stapling or suturing for anastomoses of the left side of the large intestine. Surg Gynecol Obstet. 1990;171(5):373-376.

19. Wang L, Gu J. Risk factors for symptomatic anastomotic leakage after low anterior resection for rectal cancer with $30 \mathrm{~Gy} / 10 \mathrm{f} / 2 \mathrm{w}$ preoperative radiotherapy. World J Surg. 2010;34(5):1080-1085. doi: 10.1007/s00268-010-0449-9.

20. McDermott FD, Heeney A, Kelly ME, Steele RJ, Carlson GL, Winter DC. Systematic review of preoperative, intraoperative and postoperative risk factors for colorectal anastomotic leaks. Br J Surg. 2015;102(5):462-479. doi: 10.1002/bjs. 9697 .

21. Crombe T, Bot J, Messager M, Roger V, Mariette C, Piessen G. Malignancy is a risk factor for postoperative infectious complications after elective colorectal resection. Int J Colorectal Dis. 2016;31(4):885-894. doi: 10.1007/s00384016-2521-x.

22. Park JS, Choi GS, Kim SH, Kim HR, Kim NK, Lee KY, et al. Multicenter analysis of risk factors for anastomotic leakage after laparoscopic rectal cancer excision: the Korean laparoscopic colorectal surgery study group. Ann Surg. 2013;257(4):665-671. doi: 10.1097/SLA.0b013e31827b8ed9.

23. Warschkow R, Steffen T, Thierbach J, Bruckner T, Lange J, Tarantino I. Risk factors for anastomotic leakage after rectal cancer resection and reconstruction with colorectostomy. A retrospective study with bootstrap analysis. Ann Surg Oncol. 2011;18(10):2772-2782. doi: 10.1245/s10434-011-1696-1.

24. Hamabe A, Ito M, Nishigori $H$, Nishizawa $Y$, Sasaki T. Preventive effect of diverting stoma on anastomotic leakage after laparoscopic low anterior resection with double stapling technique reconstruction applied based on risk stratification. Asian J Endosc Surg. 2018;11(3):220-226. doi: 10.1111/ases.12439.

25. Pakkastie TE, Luukkonen PE, Jarvinen HJ. Anastomotic leakage after anterior resection of the rectum. Eur J Surg. 1994;160(5):293-297; discussion 299-300.

\section{Author`s Contribution:}

SC conceived and designed the study.

SC, BH and XZ did data collection and manuscript writing.

SC and XZ did statistical analysis.

SC, BH and $\mathrm{XZ}$ did review and final approval of manuscript. 